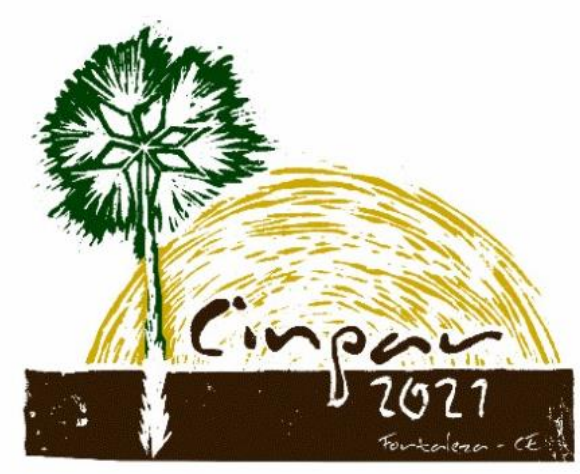

XVII Congresso Internacional sobre Patologia e Reabilitação das Construções

XVII Congreso Internacional sobre Patología y Rehabilitación de las Construcciones

XVII International Conference on Pathology and Constructions Rehabilitation

FORTALEZA (Brasil), 3 a 5 de junho de 2021

https://doi.org/10.4322/CINPAR.2021.100

\title{
Evaluación de la resistencia a la compresión de bloques de suelo-cemento con la incorporación de Ceniza de Cáscara de Arroz (CCA)
}

\section{Evaluation of the compressive strength of soil-cement blocks with the incorporation of Rice Husk Ash (RHA)}

\author{
Joaquin Humberto Aquino ROCHA ${ }^{1}$, Marialaura HERRERA-ROSAS², Nahúm Gamalier CAYO-CHILENO ${ }^{3}$, \\ Giovana Silvia CACHACA-TAPIA ${ }^{4}$, Juan Lucio BAUTISTA-ARRATIA ${ }^{5}$, Adrian PATIÑO ${ }^{6}$

\footnotetext{
${ }^{1}$ Universidad Privada del Valle, Tiquipaya, Bolivia, joaquinaquinorocha@gmail.com

${ }^{3}$ Universidad Privada del Valle, Tiquipaya, Bolivia, nahum.cayo.chileno@gmail.com

${ }^{4}$ Universidad Privada del Valle, Tiquipaya, Bolivia, g.cachacatapia@gmail.com

${ }^{5}$ Universidad Privada del Valle, Tiquipaya, Bolivia, juanluciobautista85@gmail.com

${ }^{6}$ Universidad Privada del Valle, Tiquipaya, Bolivia, adrianpspato@hotmail.com
} \\ 2 Universidad Privada del Valle, Tiquipaya, Bolivia, marialaura.herrerarosas@gmail.com
}

Resumen: El objetivo de la presente investigación es evaluar el uso de la Ceniza de Cáscara de Arroz (CCA) como reemplazo del cemento Portland (CP) en la elaboración de bloques de suelo-cemento, específicamente en la resistencia a compresión. La metodología consistió en la elaboración de bloques de suelo-cemento con tres dosificaciones: $10 \% \mathrm{CP}+0 \% \mathrm{CCA}(\mathrm{D} 1) ; 7.5 \% \mathrm{CP}+2.5 \%$ CCA (D2), y $5 \% \mathrm{CP}+5 \%$ CCA (D3). Después de un periodo de curado y endurecimiento, se determinó la resistencia a la compresión para tres edades $(7,14$ y 28 días), utilizando la media de cuatro unidades. Los resultados se compararon con la norma boliviana (NB 1211003) y la norma brasileña (NBR 10834). El reemplazo de CCA por CP reduce la resistencia a compresión en $12.37 \%$ y $27.19 \%$ para los bloques D2 y D3, respectivamente. No obstante, todos los bloques cumplen con los límites recomendados a diferentes edades. Si bien se atienden los requerimientos, el reemplazo de CCA por CP tiene tendencia negativa, por lo cual se recomienda realizar una mayor cantidad de ensayos para obtener porcentajes óptimos de sustitución sin comprometer la resistencia a la compresión.

Palabras clave: Sustentabilidad, propiedades mecánicas, viviendas sociales.

\begin{abstract}
The objective of this research is to evaluate the use of Rice Husk Ash (RHA) as a replacement for Portland cement (CP) in the elaboration of soil-cement blocks, specifically in terms of resistance to compression. The methodology consisted in the elaboration of soil-cement blocks with three dosages: $10 \% \mathrm{CP}$ + 0\% CCA (D1); $7.5 \% C P+2.5 \% C C A(D 2)$, and 5\% CP + 5\% CCA (D3). After a period of curing and hardening, the compressive strength was determined for three ages (7, 14 and 28 days), using the average of four units. The results were compared with the Bolivian standard (NB 1211003) and the Brazilian standard (NBR 10834). The replacement of RHA by CP reduces the compressive strength by $12.37 \%$ and $27.19 \%$ for blocks D2 and D3, respectively. However, all blocks meet the recommended limits at different ages. Although the requirements are met, the replacement of RHA by CP has a negative tendency, therefore it is recommended to carry out a greater number of tests to obtain optimal percentages of substitution without compromising the compressive strength.
\end{abstract}

Keywords: Sustainability, mechanical properties, social housing. 


\section{Introducción}

La vivienda es una necesidad básica del ser humano; sin embargo, representa un problema actual debido al elevado costo de los materiales y la falta de recursos, más aún en países en vías de desarrollo. Esto ha llevado a la búsqueda de nuevas alternativas frente a los materiales convencionales; opciones que puedan ser fabricadas fácilmente y a partir de recursos locales disponibles, dando lugar a una construcción sustentable (Rahman, 1988; Sekhar y Nayak 2018). Nshimiyimana et al. (2019) señalan que los materiales locales y no convencionales pueden cumplir con los propósitos de la necesidad de vivienda.

Entre los materiales de construcción sustentable, se destaca el suelo, material abundante y ampliamente utilizado (James et al., 2016). Varias investigaciones demuestran que, los materiales basados en suelo, son adecuados para la construcción de viviendas, a pesar de haber experimentado varios cambios con el tiempo (Bruno et al., 2017; Sore et al., 2018; Masuka et al., 2018).

De las técnicas que involucran el uso de suelo en la construcción, los bloques de suelo-cemento han experimentado popularidad, esto debido a sus ventajas: se constituyen de materiales ecológicos, económicamente competitivos, disponibilidad de material, facilidad de uso, resistencia al fuego, rendimiento climático beneficioso, bajo consumo de energía; no obstante, también se reportan algunas limitaciones respecto a su durabilidad, resistencia a la tracción, resistencia al impacto, resistencia a la abrasión y aceptabilidad (Villamizar et al., 2012).

Desde hace más de 50 años que los bloques de suelo-cemento se utilizan como mampostería. Su producción es sencilla, comprende la mezcla de tierra húmeda y un estabilizador, para luego pasar por una prensa (Walker, 1995; Sekhar y Nayak, 2018). Entre los estabilizantes más utilizados se encuentran: el cemento, cal, yeso, betún, puzolana, geopolímeros, entre otros (Walker, 1995; Sore et al., 2018; Masuka et al., 2018).

El cemento Portland es el estabilizador más común para la producción de bloques de suelo-cemento (Hossain y Mol, 2011; Basha et al., 2005). Se requieren alrededor de 28 días de curado para alcanzar la madurez óptima de hidratación; mientras que, otros materiales requieren mayor tiempo de curado para alcanzar rendimientos satisfactorios de resistencia y durabilidad, tal es el caso de la cal (Reddy y Hubli, 2002; Nshimiyimana et al., 2019).

No obstante, la producción de cemento emite $\mathrm{CO}_{2}$ en grandes cantidades. Pero, la utilización del cemento puede ser reemplazada con otros materiales de desecho, como la ceniza del bagazo de caña de azúcar (Alavéz-Ramírez et al., 2012; James et al., 2016), ceniza de la cáscara de arroz (Habeeb y Mahmud, 2010; Jittin et al., 2020), entre otros, proporcionando beneficios socioeconómicos (Nshimiyimana et al., 2019).

Se producen aproximadamente 100 millones de toneladas de cáscara de arroz en el mundo (Roy, 2014). Un subproducto de la cáscara de arroz es la ceniza, utilizada como un material en la construcción civil, incluida la estabilización de suelos (Jittin et al., 2020). La ceniza de cáscara de arroz (CCA) tiene un alto contenido de sílice $\left(\mathrm{SiO}_{2}\right)$ y otros óxidos, pudiendo ser utilizada como puzolana (Jittin et al., 2020). Si el contenido de sílice amorfa es elevado, puede reaccionar fácilmente con el hidróxido de calcio $\left(\mathrm{Ca}(\mathrm{OH})_{2}\right)$, este último se libera durante el endurecimiento del cemento para formar un compuesto de cementación (Roy, 2014). En este sentido, el presente estudio tiene como objetivo evaluar la influencia de la CCA como reemplazo al cemento Portland (CP) en la resistencia a la compresión de bloques de suelo-cemento.

\section{Metodología}

Se elaboraron bloques de suelo-cemento incluyendo CCA, considerando tres dosificaciones: $\mathrm{D} 1,10 \% \mathrm{CP}+0 \%$ CCA; D2, 7.5\% CP + 2.5\% CCA, 5\% CP + 5\% CCA. Se recomienda que el contenido de cemento sea mayor al $4 \%$, esto para obtener buenos resultados en las propiedades de resistencia y durabilidad (Tripura y Singh, 2015). Las dimensiones del bloque fabricado se presentan en la Figura 1.

Se utilizó un cemento Portland comercial IP-30, clasificado como cemento puzolánico de acuerdo a la norma ASTM 595 (ASTM, 2020). El Cuadro 1 presenta el análisis químico del cemento.

La CCA fue recolectada de un ingenio arrocero de la región de Santa Cruz, Bolivia. Siguiendo las recomendaciones de la literatura (Jittin et al., 2020), la CCA fue sometida a un proceso de combustión 
controlada, 700 a $800^{\circ} \mathrm{C}$, para obtener sílice amorfa. Posteriormente la CCA pasó el tamiz №200. La Figura 2 muestra la CCA antes y después del tamizado.

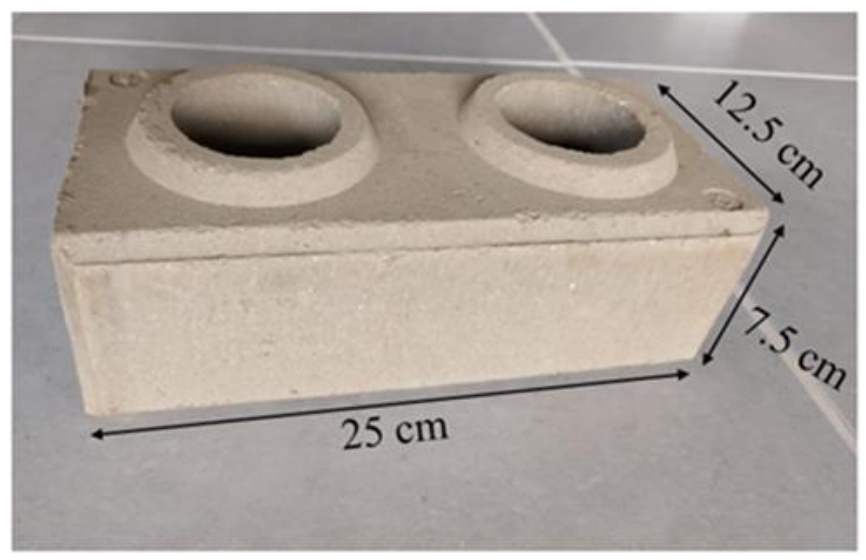

Figura 1 - Dimensiones del bloque elaborado

Cuadro 1 - Análisis químico del cemento utilizado

\begin{tabular}{|c|c|c|}
\hline Parámetro & Unidad & Cemento IP-30 \\
\hline Pérdida por ignición & $\%$ & 2.33 \\
\hline $\mathrm{SiO}_{2}$ & $\%$ & 32.83 \\
\hline $\mathrm{Al}_{2} \mathrm{O}_{3}$ & $\%$ & 4.53 \\
\hline $\mathrm{Fe}_{2} \mathrm{O}_{3}$ & $\%$ & 2.32 \\
\hline $\mathrm{CaO}$ & $\%$ & 50.77 \\
\hline $\mathrm{MgO}$ & $\%$ & 4.55 \\
\hline $\mathrm{SO}_{3}$ & $\%$ & 2.10 \\
\hline
\end{tabular}

Datos proporcionados por el fabricante
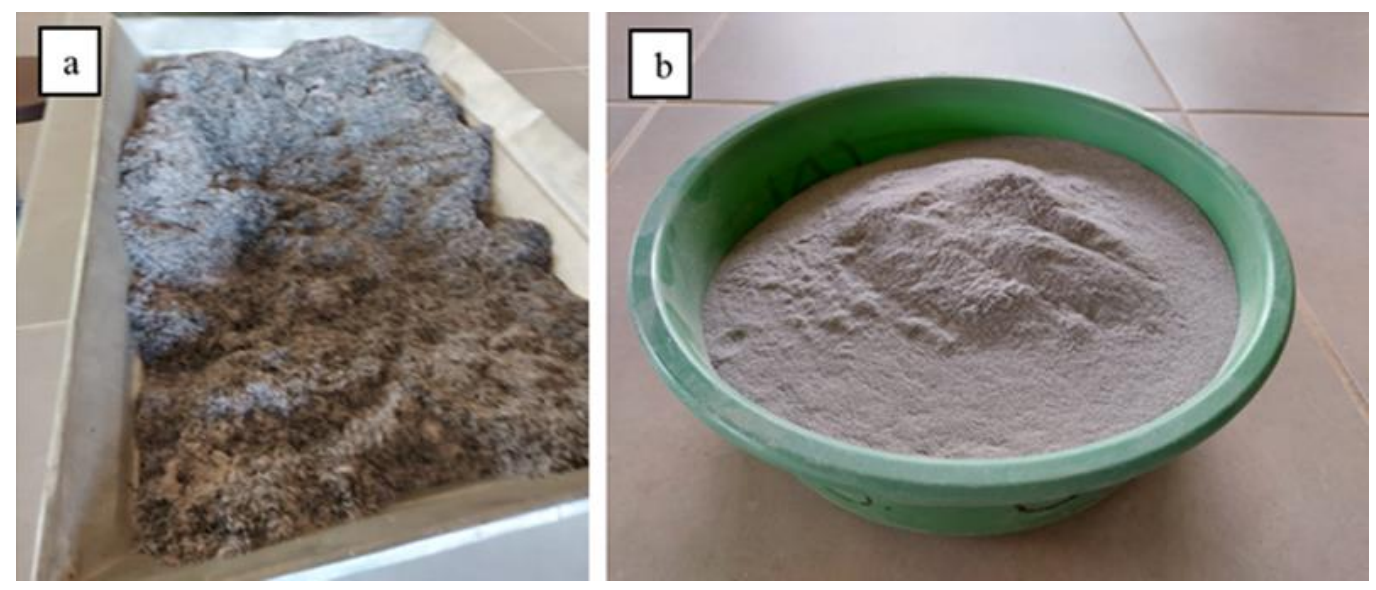

Figura 2 - CCA: a) antes y b) después de pasar por el tamiz $N^{\circ} 200$

Mediante el ensayo de Fluorescencia de Rayos X (FRX) se determinó la composición química de la CCA utilizada (Cuadro 2).

El suelo fue extraído de la zona sur de la ciudad de Cochabamba. Donde se realizaron los ensayos de: granulometría, mediante la norma D422 (ASTM, 2007); límites de Atterberg, usando la norma D4318 (ASTM, 2017a), y el ensayo de compactación estándar Proctor, de acuerdo con la norma D698 (ASTM, 2012). 
Cuadro 2 - Análisis químico del cemento utilizado

\begin{tabular}{|c|c|c|}
\hline Componente & Unidad & CCA \\
\hline $\mathrm{SiO}_{2}$ & $\%$ & 90.74 \\
\hline $\mathrm{K}_{2} \mathrm{O}$ & $\%$ & 0.87 \\
\hline $\mathrm{Al}_{2} \mathrm{O}_{3}$ & $\%$ & 0.09 \\
\hline $\mathrm{Fe}_{2} \mathrm{O}_{3}$ & $\%$ & 0.32 \\
\hline $\mathrm{CaO}$ & $\%$ & 1.01 \\
\hline $\mathrm{MgO}$ & $\%$ & 0.43 \\
\hline $\mathrm{Na} 2 \mathrm{O}$ & $\%$ & 0.33 \\
\hline
\end{tabular}

Para mezclar los componentes de los bloques (CP, CCA, suelo y agua), se utilizó una mezcladora mecánica. Posteriormente con la ayuda de una máquina compresora tipo lego, se elaboraron los bloques de manera manual.

Siguiendo las recomendaciones de Gatani (2000), los bloques de suelo-cemento pasaron por un proceso de curado de 7 días (regado con agua) y se mantuvieron en laboratorio hasta el día del ensayo.

Los bloques se sometieron a ensayos de resistencia a la compresión para la edad de 7, 14 y 28 días, tomando en cuenta las recomendaciones de la norma NBR 8492 (ABNT, 2012). Se utilizó el promedio de cuatro bloques para cada edad y dosificación, totalizando 36 bloques ensayados.

\section{Resultados}

\subsection{Suelo utilizado}

La curva granulométrica del suelo ensayado se observa en la Figura 3; el porcentaje de arena es $65 \%$ y de los limos y arcillas 35\%, aproximadamente.

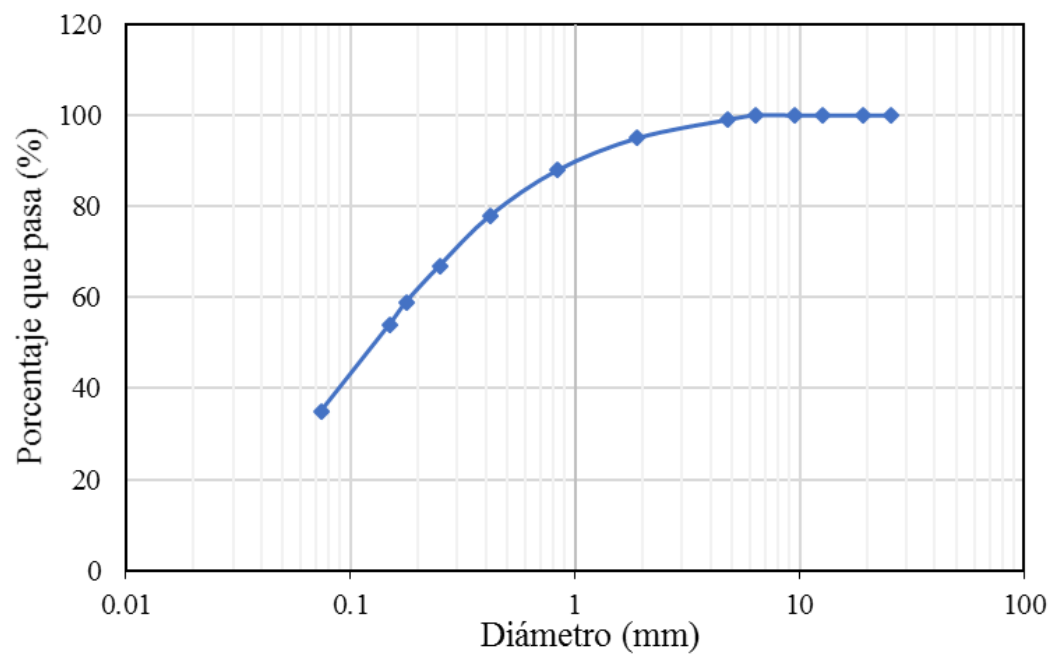

Figura 3 - Curva granulométrica del suelo utilizado

El Límite Líquido (LL) y el Índice Plástico (IP) son 24 y 10, respectivamente. El suelo se clasifica como arena arcillosa (SC) de acuerdo al Sistema Unificado de Clasificación de Suelos (SUCS) (ASTM, 2017b).

Con respecto al ensayo de compactación estándar Proctor (Figura 4), se observa que, en las dosificaciones con CP y CCA, la densidad seca decrece y el contenido de humedad óptima aumenta a comparación de dosificación sin estabilizante. Basha et al. (2005) también reportaron esta misma tendencia. 


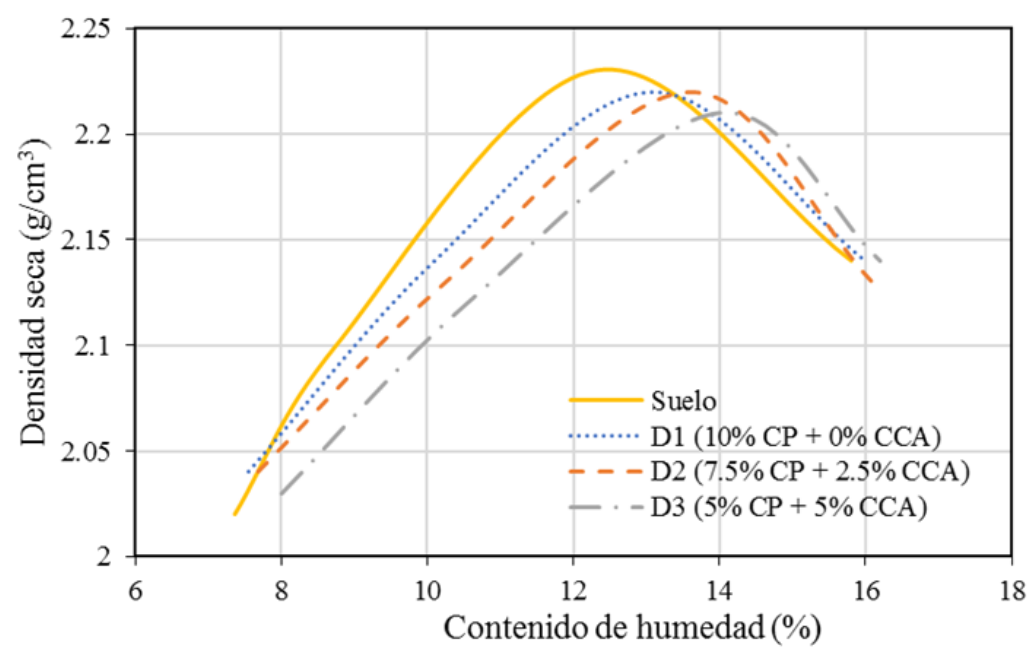

Figura 4 - Ensayo de compactación estándar Proctor

\subsection{Resistencia a la compresión}

La Figura 5 muestra los resultados de resistencia a la compresión de los bloques ensayados. Se observa que, los bloques D1, tienen en promedio mayor resistencia a la compresión que los bloques D2 y D3, para todas las edades.

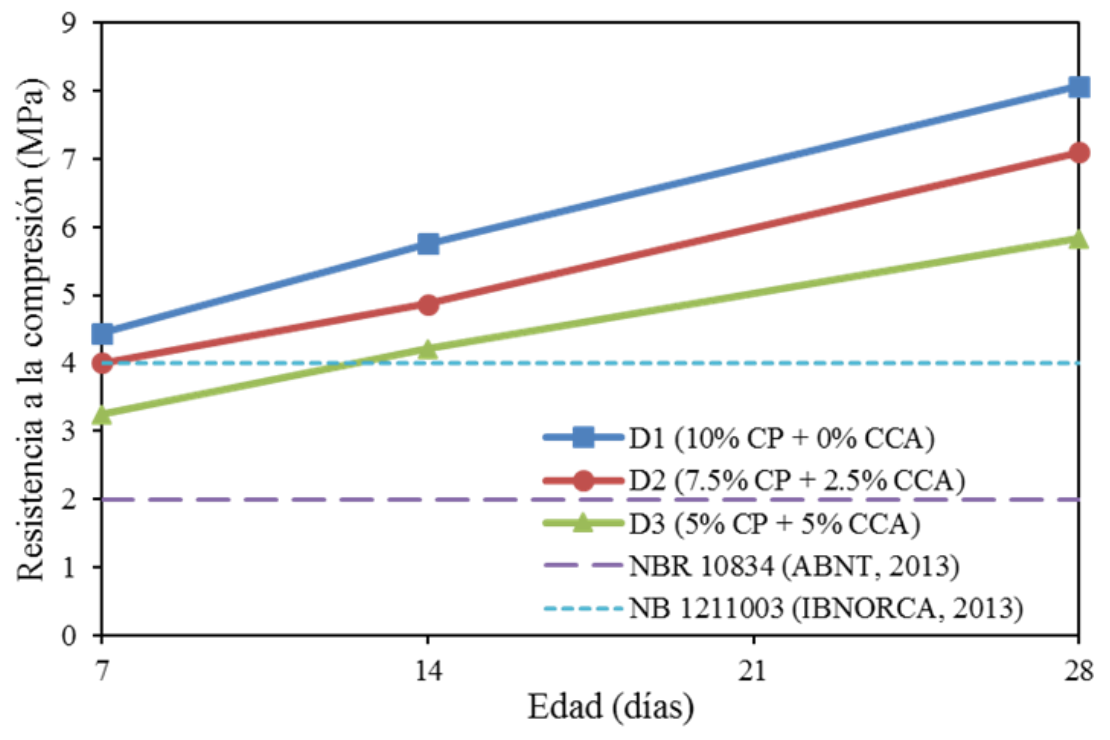

Figura 5 - Resistencia a la compresión

Si bien no existe normativa de bloques de suelo-cemento en Bolivia, se utilizó la NB 1211003 (IBNORCA, 2013) de ladrillos cerámicos a fin de comparar los resultados. Dentro de esta normativa, los bloques D1 y D2 corresponden a una categoría $\mathrm{C}$, aptos para construcciones de uso general; mientras que los bloques D3 entran a esta categoría a partir de los 14 días.

La NBR 10834 (ABNT, 2013) de bloques de suelo cemento sin función estructural, indica que el promedio de la resistencia a la compresión debe ser mayor a $2 \mathrm{MPa}$ y que cada valor individual debe estar por encima de 1.7 $\mathrm{MPa}$, para todos los casos se cumplieron estas recomendaciones.

La Figura 6 presenta la reducción porcentual de la resistencia a la compresión en comparación a los bloques D1. Existe una reducción de $12.37 \%$, en promedio, cuando se reemplaza el $2.5 \%$ de CP por CCA; mientras que, si el reemplazo es del $5 \%$ del CP por CCA, se tiene $27.19 \%$ de reducción. 


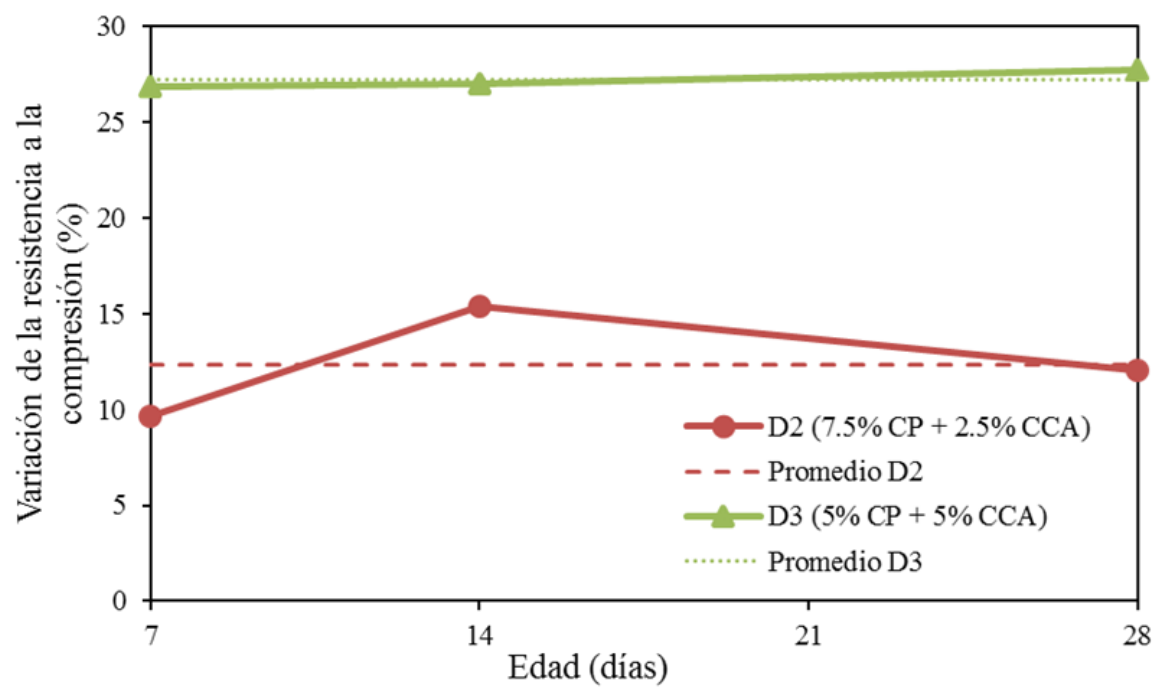

Figura 6 - Resistencia a la compresión

Si bien estos porcentajes de variación son considerables, los valores se encuentran dentro de los límites recomendados.

\section{Conclusiones}

El presente estudio presenta una alternativa sustentable en la construcción civil; la utilización de residuos, en este caso la ceniza de cascara de arroz, en sustitución parcial del cemento Portland.

Se demostró que la producción de bloques suelo-cemento con CCA en reemplazo al CP, es viable, puesto que los resultados de la resistencia a la compresión fueron favorables a distintas edades, atendiendo a los límites recomendados por la normativa boliviana y brasilera.

A pesar de que los resultados son positivos, existe reducción en la resistencia a la compresión; por tanto, se deben encontrar porcentajes óptimos para la utilización de CCA. Todavía es necesario realizar investigaciones posteriores que involucren ensayos de durabilidad, resistencia a la flexión, resistencia a la tracción, entre otros, para consolidar su aplicación.

\section{Referencias Bibliográficas}

ABNT (2012). NBR 8492: Tijolo de solo cimento - Análise dimensional, determinação da resistência a compressão e da absorção de água - Método de ensaio. Associação Brasileira de Normas Técnicas (ABNT), Rio de Janeiro

ABNT (2013). NBR 10834: Bloco de solo-cimiento sem funçã estructural - Requisitos. Associação Brasileira de Normas Técnicas (ABNT), Rio de Janeiro.

Alavéz, R., Montes, P., Martínez, J., Altamirano, D.C., Gochi, Y. (2012). The use of sugarcane bagasse ash and lime to improve the durability and mechanical properties of compacted soil blocks. Construction and Building Materials, 34, 296-305.

ASTM (2007). ASTM D422-63(2007)e2: Standard Test Method for Particle-Size Analysis of Soils (Withdrawn 2016). ASTM International, West Conshohocken, PA.

ASTM (2012). ASTM D698-12e2: Standard Test Methods for Laboratory Compaction Characteristics of Soil Using Standard Effort (12 $400 \mathrm{ft}-\mathrm{lbf} / \mathrm{ft} 3(600 \mathrm{kN}-\mathrm{m} / \mathrm{m} 3))$, ASTM International, West Conshohocken.

ASTM (2017a). ASTM D4318-17e1: Standard Test Methods for Liquid Limit, Plastic Limit, and Plasticity Index of Soils, ASTM International, West Conshohocken, PA.

ASTM (2017b). ASTM D2487-17e1: Standard Practice for Classification of Soils for Engineering Purposes (Unified Soil Classification System), ASTM International, West Conshohocken, PA. 
ASTM (2020). ASTM C595/C595M-20: Standard Specification for Blended Hydraulic Cements. ASTM International, West Conshohocken, PA.

Basha, E.A., Hashim, R., Mahmud, H.B., Muntohar, A.S. (2005). Stabilization of residual soil with rice husk ash and cement. Construction and Building Materials, 19, 448-453.

Bruno, A.W., Gallipoli, D., Perlot, C., Mendes, J. (2017). Mechanical behaviour of hypercompacted earth for building construction. Materials and Structures, 50.

Gatani, M.P. (2000). Ladrillos de suelo-cemento: mampuesto tradicional en base a un material sostenible. Informes De La Construcción, 51(466), 35-47.

Habeed, G.A., Mahmud, H.B. (2010). Study on propeties of rice husk ash and it use as cement replacement material. Materials Research, 13(2), 185-190.

Hossain, K.M.A., Mol, L. (2011). Some engineering properties of stabilized clayey soils incorporating natural pozzolans and industrial wastes. Construction and Building Materials, 25(8), 3495-3501.

IBNORCA (2013). NB 1211003: Ladrillos cerámicos - Ladrillos macizos - Clasificación y requisitos (Tercera revisión). Instituto Boliviano de Normalización y Calidad (IBNORCA), La Paz.

James, J., Padian, P.K., Deepika, K., Manikanda, J., Manikandan, V., Manikumaran, P. (2016). Cement Stabilized Soil Blocks Admixed with Sugarcane Bagasse Ash. Journal of Engineering, 2016, 1-9.

Jittin, V., Bahurudeen, A., Ajinkya, S.D. (2020). Utilisation of rice husk ash for cleaner production of different construction products. Journal of Cleaner Production, 263121578.

Masuka, S., Gwenzi, W., Rukuni, T. (2018). Development, engineering properties and potential applications of unfired earth bricks reinforced by coal fly ash, lime and wood aggregates. Journal of Building Engineering, 18, 312-320.

Nshimiyimana, P., Messan A., Zhao, Z., Courard, L. (2019). Chemico-microstructural changes in earthen building material containing calcium carbide residue and rice husk ash. Construction and Building Materials, 216, 622-631.

Omar, S., Messan, A., Prud'homme, E., Escadeillas, G., Tsobnang, F. (2018). Stabilization of compressed earth blocks (CEBs) by geopolymer binder based on local materials from Burkina Faso. Construction and Building Materials, 165, 333-345.

Rahman, M.A. (1988). Effect of rice husk ash on the properties of bricks made from fired lateritic soil-clay mix. Materials and Structures, 21(3), 222-227.

Roy, A. (2014). Soil stabilization using rice husk ash and cement. International journal of civil engineering research, 5(1), 49-54.

Sekhar, C.D., Nayak, S. (2018). Utilization of granulated blast furnace slag and cement in the manufacture of compressed stabilized earth blocks. Construction and Building Materials, 166, 531-536.

Tripura, D.D., Singh, K.D. (2015). Characteristic Properties of Cement-Stabilized Rammed Earth Blocks. Journal of Material in Civil Engineering, 27(7), 04014214.

Venkatarama, B.V., Hubli, S.R. (2002). Properties of lime stabilised steam-cured blocks for masonry. Materials and Structures, 35, 293 (2002).

Villamizar, M.C.N., Araque, V.S., Reyes, C.A.R., Silva, R.S. (2012). Effect of the addition of coal-ash and cassava peels on the engineering properties of compressed earth blocks. Construction and Building Materials, 36, 276-286.

Walker, P.J. (1995). Strengrh, durability and shrinkage characteristics of cement stabilised soil blocks. Cement and Concrete Composites, 17 (4), 301-310. 